\title{
An Approach for Selective Beacon Forwarding to Improve Cooperative Awareness
}

\author{
Robert K. Schmidt ${ }^{\S}$, Robert Lasowski*, Tim Leinmüller ${ }^{\S}$, Claudia Linnhoff-Popien*, and Günter Schäfer ${ }^{\ddagger}$ \\ $\S$ DENSO AUTOMOTIVE Deutschland GmbH, Germany, \{r.schmidt|t.leinmueller\}@denso-auto.de \\ *Institute for Mobile and Distributed Systems, Ludwig Maximilian University of Munich, Germany, \\ \{robert.lasowski|linnhoff\}@ifi.lmu.de \\ ${ }^{\ddagger}$ Telematics/Computer Networks Research Group, Technical University of Ilmenau, Germany, \\ guenter.schaefer@tu-ilmenau.de
}

\begin{abstract}
Cooperative awareness is established by vehicles exchanging their status frequently. In situations where a high number of vehicles access the communication channel with high frequency, communication and cooperative awareness suffer from increased packet loss.

So far, forwarding of beacons focuses on extending the range of $\mathrm{V} 2 \mathrm{~V}$ communication which is not necessarily needed in most scenarios. In this paper, we motivate selective beacon forwarding to improve the reliability of cooperative awareness in high load situations. We define a metric to measure the quality of cooperative awareness and compare different static beacon rates by a simulation study. Especially in high load situations, we evaluate selective forwarding of beacons to overcome the awareness degradation due to interference, leading to packet loss even at short distances. We show that our approach causes only slight overhead in terms of additional messages and that the age of forwarded information is less than half the beacon interval.
\end{abstract}

\section{INTRODUCTION}

The continuous improvement of road safety has always been an important objective within the automotive area. Therefore advanced driver assistance systems based on vehicular adhoc networks (VANET) are in the focus of the current research activities. These systems shall enhance drivers' horizon to reduce the reaction time in case of road situations that could potentially lead to traffic accidents. Thus, vehicles are equipped with wireless communication technology, operating according to the standard IEEE $802.11 \mathrm{p}$.

Vehicles exchange two types of messages to establish awareness of each other: Periodically transmitted beacon messages which are also referred to as Cooperative Awareness Messages (CAM) [1] and event-driven Decentralized Environmental Notification messages (DENM) |2|.

Beacons basically contain the state of a vehicle, like the current vehicle position. Due to the highly dynamic network topology, beacon messages have to be sent with high frequency to ensure up-to-date information and mitigate single packet loss. However, frequently sending messages leads to a high channel load and potential collisions on the communication channel which in the end results in a low reception probability and information loss. This is especially the case in traffic scenarios of high density, where the channel can be overloaded. In |3|, we identified that the overload of the channel causes packet loss occurring at larger distances between sender and receiver. At shorter distances, we observed a certain area of very low packet loss, nearly independent of the channel load, i.e. the communication range under interference. Furthermore, spatio-temporal packet loss occurs due to significant shadowing by stationary and non-stationary obstacles like buildings or heavy trucks, a known problem of communication in nonline-of-sight (NLOS) $|4|$ between transmitter and receiver.

In this paper, we elaborate on selective beacon forwarding which addresses spatio-temporal packet loss due to shadowing and leverages from the reliable communication range in order to circumvent packet loss in high load situations. In the design of the approach we carefully take application-level requirements into account. Mak et al. discuss in [5] that for cooperative collision and intersection warning applications, up-to-date information must be available in a range between 50 and $300 \mathrm{~m}$ around the transmitting vehicle. This range is much lower than the possible communication range of $1 \mathrm{~km}$. Thus, we apply forwarding to improve the awareness within the application-relevant area instead of extending the singlehop range.

The paper is structured as follows. Sec. II surveys existing forwarding approaches and motivates our work guided by the Design Goals and Metrics given in Sec. III. Our approach is developed in Sec. IV and its performance regarding line-ofsight is evaluated in Sec. V. We conclude in Sec. VI

\section{RELATED WORK}

The improvement of vehicles' awareness by selectively forwarding of beacons has not been studied in the literature so far. Most of the existing approaches related to forwarding of messages consider feedback from single nodes and retransmissions as a solution to increase the range of broadcast communication over multiple hops.

Korkmaz presents the idea of a reliable urban multihop broadcast protocol in [6]. This protocol refers to the RTS/CTS handshake from the IEEE 802.11 standard and introduces a Request to Broadcast (RTB) packet. After receiving an RTB, a vehicle answers with a channel jamming signal whose duration depends on the distance to the source of the RTB. The vehicle with the highest distance transmits the longest jamming signal. After transmission, this vehicle will find the channel clear and is considered as the selected forwarder. This algorithm aims at message dissemination over long distance and does not meet the requirement of cooperative safety applications. 
Seungjin et al. [7] propose a one-hop algorithm which is based on negative acknowledgments (NAK). The authors propose to send NAK messages if any node detects a packet collision which triggers a retransmission of the message from the source node. This process is performed until the message received by every node. The original message is considered to received by all surrounding vehicles if no NAK messages is received by the source node. However, the proposed algorithm will not terminate if the NAK message itself collides on the communication channel or if the source vehicle has moved out of range meanwhile.

Mariyasagayam et al. [8] propose Multi-Hop Vehicular Broadcast (MHVB), a contention-based beacon forwarding mechanism. Based on the distance a vehicle calculates a contention time that defines the time the vehicle has to wait before forwarding a message. Thus, vehicles with a high distance are preferred to be the next forwarder. MHVB further defines a so called backfire algorithm to suppress additional forwarding of messages in case it has already been forwarded.

Based on the idea of MHVB, Mittag et al. study in [9] the multi-hop forwarding approach in combination with transmit power reduction and compare it with plain single-hop communication. Metrics are defined to evaluate the channel load by the number of data offered by the vehicles, neighborhood awareness and beacon information age. The paper concludes that multi-hop forwarding of all beacons is not able to provide the same awareness as single-hop forwarding with higher transmit power. However, it is left open how the awareness can be increased to nearly 100 percent, as demanded by active safety applications. Especially, a study of higher dense scenarios under significant interference and packet loss would be needed to evaluate awareness degradation, occurring even in the application-relevant area.

Saleh et al. [10] consider the reliability as main concern for Quality of Service (QoS) in VANETs. The communicationlevel QoS and application-level QoS may not necessarily be the same and recommend to take the application requirements more in to account while designing new protocols. The authors introduce a new metric named effective range which considers requirements from a safety application. It evaluates in which range a pre-defined set of QoS requirements can be satisfied.

In general the proposed algorithms concentrate on forwarding to improve routing. Especially high overhead is added to the communication channel and poses high latency in message dissemination if all beacons are forwarded. We emphasize that a careful forwarding of beacons within a smaller, pre-defined range is a valuable means to improve reliability instead of extending the transmission range. Therefore, we propose a new metric quantifying the awareness quality needed for collision avoidance applications.

\section{Design GoAls And Metrics}

In this section, we discuss general design goals for an appropriate forwarding approach that improves the quality of cooperative awareness, efficiently and cooperatively uses the communication channel, and is able to operate properly even under high channel load. Therefore, we establish dedicated metrics for an appropriate evaluation and derive refined design goals.

\section{A. Quality of Cooperative Awareness}

Periodically exchanging beacon messages establishes upto-date awareness of all surrounding vehicles and their status. Most important is the safety-relevant area of 50 to 300 meters around the vehicle, depending on the velocities. Thus, in this area we consider the quality of cooperative awareness, consisting of two characteristics: The awareness quantile and the age of received forwarded information.

1) Awareness Quantile: We define awareness as the relation between knowledge of vehicles that is stored in a vehicle's neighbor table and the knowledge of vehicles that should be stored. The neighbor table is created from information obtained via beacon messages.

In contrast to [9], we define the awareness metric from the perspective of each vehicle's knowledge. For a given quantile $\alpha$, each vehicle measures if it has a certain awareness quantile or not. For example, for an awareness quantile of $\alpha=90 \%$, each vehicle determines if it has up-to-date information of at least $90 \%$ of the surrounding vehicles, stored in the neighbor table. Taking into consideration the application-relevant distance $d$, we can define the metric as follows.

$\mathcal{V}^{d}$ denotes the set of all vehicles within a distance $d$. The set of all discovered neighbors is denoted as $\mathcal{N}^{d}$. A neighbor is deleted from the neighbor table, once the previously received beacon becomes outdated. At time $t$ and for a certain vehicle $i$, we can establish the awareness quantile as follows:

$$
\text { Awareness }_{\alpha, d, t}(i)= \begin{cases}1 & \frac{\left|\mathcal{N}_{i}^{d}(t)\right|}{\left|\mathcal{V}_{i}^{d}(t)\right|}>\alpha \\ 0 & \text { else }\end{cases}
$$

There are various reasons why this ratio can be less than 1, for example a low penetration rate degrades this ratio significantly. However in this article, we focus only on communication aspects. First, shadowing of objects has a strong influence on the signal attenuation which may result in packet loss. Second, especially in high load situations, packet loss occurs due to interference. The packet loss may even occur at low distances between sender and receiver which would most likely prevent active safety applications to work properly.

In order to measure the Awareness Quantile (AQ) over time, the awareness is summed up over all vehicles and divided by all vehicles.

$$
A Q=\frac{\sum_{i \in \mathcal{V}} \text { Awareness }_{\alpha, d, t}(i)}{|\mathcal{V}|}
$$

For the rest of the paper, we will only consider the AQ with $\alpha=100 \%$, the most strict threshold. This will emphasize even minor reductions of the awareness which may be severe in case of a safety-related incident.

2) Age of Received Forwarded Information: Active safety applications typically require a transmission latency of less than $100 \mathrm{msec}$ [11]. Single-hop broadcast communication usually does not violate this requirement. However, two or 
multi-hop communication easily multiplies the single-hop delay of some milliseconds. Therefore, we define the age of received forwarded information as the time difference between originally sending the information and finally receiving it from a forwarder.

\section{Age $=$ ForwardReceivedTime - BeaconGenerationTime}

The age can be increased beyond multiples of the transmission delay, for example if beacons are not immediately forwarded. Thus, inefficient forwarding has to be avoided by selective forwarding so that nearly outdated information is not forwarded.

\section{B. Efficiency}

Cooperative awareness has to be established efficiently and improved cooperatively. As the awareness is basically created using a static beacon rate, an analysis of different beacon rates is necessary, extending the analysis in $|3|$. As loss of information in VANET communication is known to occur under high channel load/interference, we employ the metric Success Rate to evaluate the efficiency of the channel usage also when forwarding of beacons is applied.

To improve the awareness cooperatively, the benefit of a forward should be as high as possible. As all vehicles have to share the same channel, it is desirable that as many vehicles as possible have a benefit from a forwarded beacon. The minimum requirement would be to achieve that at least one vehicle benefits from a forward. Hence, we evaluate how and how much the vehicles benefit from a forwarded beacon.

1) Success Rate: In the best case, if there is negligible interference and all transmissions are perfectly aligned in time without overlap, the Success Rate would be 1. In other words, all received packets with sufficient absolute power can be decoded. With interference on the channel, the signal-to-noiseinterference ratio is not high enough to decode the packet. So, the lower the ratio, the higher the impact of interference:

$$
\text { SuccessRate }=\frac{\text { Number of decoded packets }}{\text { Number of received strong signals }} .
$$

Note that this metric can only be measured in the simulation, where all signals can be separated from each other.

2) Primary and Secondary Benefit: Vehicles that receive a forwarded beacon which they have not received before may have two different types of benefit.

The primary benefit is achieved when a receiving vehicle updates its awareness database triggered by a forwarded beacon.

$$
\text { PrimaryBenefit }=\frac{\text { Number of neighbor updates }}{\text { Number of sent forwards }} .
$$

The secondary benefit is counted when a received forwarded beacon adds a new neighbor to awareness database.

$$
\text { SecondaryBenefit }=\frac{\text { Number of new neighbors }}{\text { Number of sent forwards }} .
$$

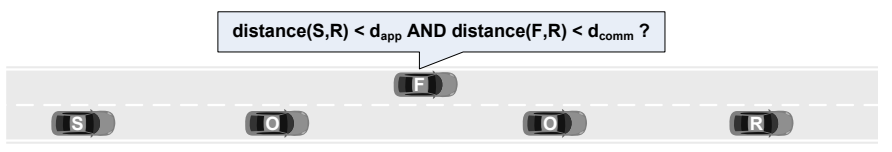

Fig. 1. Forwarding decision: $F$ forwards beacon from $S$ because $R$ requested it and distance relations are fulfilled.

To improve the quality of awareness, the selective forward should aim at increasing the primary benefit. The secondary benefit underlines the efficiency of the approach, i.e. not to transmit useless information.

\section{Robustness}

As the communication for safety-related applications is prone to the overload of the channel, a selective forwarding of beacons should not deteriorate the communication. Thus, the Success Rate should not be significantly degraded compared to the regular exchange of beacons. A selective and cautious forwarding of beacons even under high load should contribute to the improvement of the awareness in the safety-relevant area with reasonable benefit. It is not desirable to initiate forwards targeting (few) vehicles at large distances which are subject to collisions and hence low benefit.

Similarly, selective forwarding should overcome changing signal propagation environments and circumvent significant spatio-temporal signal attenuation, resulting from shadowing by obstacles like buildings or trucks.

\section{Selective Forwarding to Improve Cooperative AWARENESS}

We base our approach on a generally-known beacon rate. This allows to detect a packet loss, using the pre-defined beacon interval plus a small time difference to tolerate a higher medium access delay. A vehicle requests a forward of a beacon, as it knows the ID from the vehicle via the previously received beacon.

Hence, the enabling strategy is that vehicles may request a forward of a beacon they have missed. To avoid additional messages besides the forward, the beacon request should be piggybacked in the regular beacon. The lower message overhead goes at the expense of the information age. Before extending and refining these strategies, we introduce the following terminology and roles.

\section{A. Terminology and Roles}

A vehicle (source $S$ ) generates and originally sends a beacon. A vehicle (requester $R$ ) piggybacks beacon update request(s). A vehicle (forwarder $F$ ) responds to the request by forwarding the beacon. Other vehicles (overhearer $O$ ) that overhear this dialogue skip the transmission of the already forwarded beacon. The application-relevant distance around a vehicle which demands the highest/maximum awareness is referred to as $d_{a p p}$. The maximum distance of the potential forwarder to the requester is denoted as $d_{\text {comm }}$ and should be in the order of the assumed communication range under interference.

The described notations and its relations are depicted in Fig. 1 


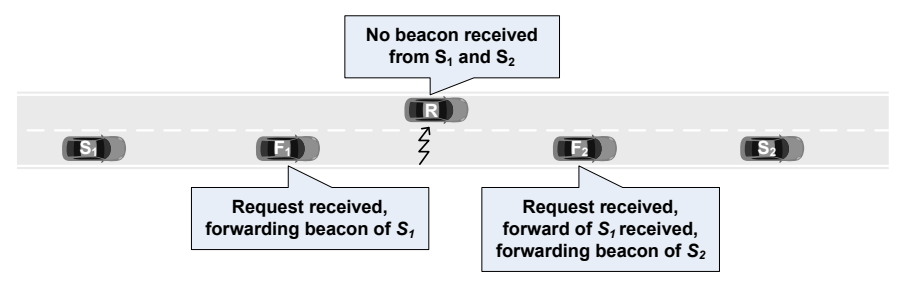

Fig. 2. $R$ did not receive beacons from $S_{1}$ and $S_{2}$ due to collision. $F_{1}$ and $F_{2}$ respond consecutively by forwarding the requested beacons.

\section{B. Forwarding strategy}

In high load situations the packet loss probability increases. Thus, we define that only vehicles within the assumed reliable communication range $d_{c o m m}$ to the requester(s) should forward a beacon.

Every potential requester $R$ checks if the beacon receive time is older than beacon interval and if the source was within an application-relevant distance $d_{a p p}$. The forward within this distance is also intended to overcome significant shadowing due to obstacles like buildings or trucks.

After receiving a request, every potential forwarder $F$ does the following. First, check if the requested beacon is available, and if the distance to $R$ is less than $d_{\text {comm }}$. Second, check if there are multiple open beacon requests. If so, concatenate multiple beacons to one forward message. Third, to avoid medium access collisions, apply a random backoff to spread the start of a transmissions among the potential forwarders. Fourth, suppress the forwarding of beacon(s) if there has already been a response by another vehicle to this request.

\section{Motivation and strategy background}

The strategy described before is expected to compensate two significant sources of packet loss: Interference and shadowing. We describe in the following how each source of packet loss is addressed by selective beacon forwarding.

1) Compensate packet loss due to interference: Fig. 2 shows an example how selective forwarding within the communication range under interference provides missing information to a requesting vehicle where the original transmissions by the two sources collided.

Due to its periodicity of the beacon, packet loss will occur periodically as the vehicles do not get any feedback from the collision and will continue to generate and transmit beacons at the same intervals. Thus, the selective forwarding approach is expected to provide a high likeliness to successfully receive the beacon from a forwarder as its transmission is spatially and timely separated from the source. To prevent periodicity in the forward, the contention phase of the potential forwarders is randomized to further avoid medium access collisions. This additional backoff is simply picked from a linear distribution. As an optimization strategy, a distance-based contention can be employed, however only if replied to one requester.

The defensive strategy of piggybacking and randomized forwarding delays goes at the expense of the information age. However, this disadvantage is assumed to decline in high vehicle densities. With a higher number of potential requesters and forwarders, the time until a forward occurs will be reduced

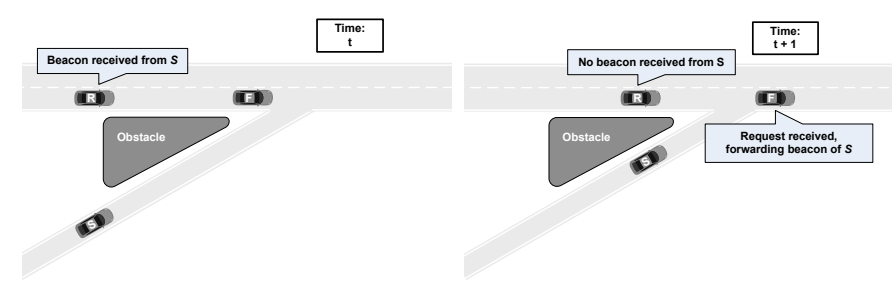

Fig. 3. $R$ missed the beacon of $S$ due to the shadowing obstacle and requests the beacon of $S$ at $t+1$. $F$ forwards the beacon.

while an increase of the benefit of the forward is expected. Some vehicles may even receive a response before sending the beacon with the piggybacked request, when another vehicle already requested the same beacon. As beneficial byproduct, the forward even increases the single-hop communication range and hence increases the awareness to two hops which we count as secondary benefit. We will investigate these hypothesis' in the following simulation study.

2) Compensate packet loss due to shadowing: Fig. 3 depicts an example how a beacon loss due to shadowing can be compensated by selective forwarding. Vehicles $S, R$ and $F$ exchange beacons at the time $t$ where line of sight between all vehicles exist. At time $t+1$ an obstacle occurs e.g. a building or a heavy truck that attenuates the received signal at $R$ which can result in packet loss at $R$. At time $t+1, R$ determines that expected beacons has not been received, yet. Thus, $R$ requests the original beacon of $S$ by sending a forward request to all their neighbors. $F$ determines if it as received the beacon of $S$ and then forwards it. Once received, $R$ has up-to-date awareness of $S$ again. Note that in this example, the roles of $R$ and $S$ can be also exchanged vice versa.

\section{Bootstrapping}

Due to long-term shadowing and/or long-term interference may prevent vehicles from receiving other vehicles being even in close distance. As a request for a beacon can only be made, after at least one beacon has been received before, a procedure is needed to get the respective vehicle ID. However, so far there is no efficient strategy to achieve this. Flooding causes the broadcast storm which we actively want to prevent. For the bootstrapping, a seldom transmission (or piggybacking) of the neighbor list including their IDs may solve this. This neighbor list must be distributed beyond the single-hop range in order to reach unknown neighbors. As such a mechanism demands another careful evaluation, we do not employ any bootstrapping for the evaluation given in the following section but will investigate this in future work.

\section{Performance Evaluation}

In the simulation study, we compare static beacon rates and the selective forward approach regarding the awareness quality, efficiency, cooperativeness and the ability to operate under high load situations.

\section{A. Simulation Scenario and Configuration}

Using the simulation framework JiST/SWANS with VANET extensions from the University of Ulm, we simulate a 


\begin{tabular}{|l|r|}
\hline Fixed Parameter & Value \\
\hline Simulation time & 60 seconds \\
\hline Number of runs & 10 \\
\hline Pathloss Model & Two-Ray Ground \\
\hline Fading Model & Rayleigh Fading \\
\hline Transmit Power & $16 \mathrm{dBm}$ \\
\hline Carrier/Receiver SINR & $5 / 8 \mathrm{~dB}$ \\
\hline Signal propagation delay & Distance-based \\
\hline Beacon delay jitter & $-1 \ldots 1 \mathrm{~ms}$ \\
\hline Noise/Interference model & Thermal/Accumulative avg power \\
\hline Maximum communication range & ₹ $1 \mathrm{~km}$ \\
\hline MAC-Layer Protocol & IEEE P802.11p \\
\hline AIFS (AC_BK) & 9 \\
\hline Contention Window & $15 \mathrm{slots}$ \\
\hline Data rate & $650 \mathrm{MBit} / \mathrm{s}$ \\
\hline Beacon length & $4 \mathrm{~km} \times 4 \mathrm{~km}$ \\
\hline Field size & Street-Random Waypoint $($ Straw $)$ \\
\hline Mobility and road model & Values \\
\hline \hline Varied Parameters & $200,400,600,800,1200,1600,2000$ \\
\hline \hline Number of vehicles & $2,4,6,8,10 \mathrm{~Hz}$ \\
\hline Beacon rate & $50,100,200,300 \mathrm{~meters}$ \\
\hline$d_{c o m m}=d_{a p p}$ & \\
\hline
\end{tabular}

TABLE I

Simulation PARAMETERS OVERVIEW

metropolitan area of $4 \mathrm{~km} \times 4 \mathrm{~km}$ with 200 to 2000 vehicles. The beacon rates under test range from 2 to $10 \mathrm{~Hz}$, as these are typical values proposed for active safety applications in VANETs.

For the selective forwarding, we simply keep both parameters equal by setting $d_{a p p}=d_{\text {comm }} \in\{50,100,200,300\}$ meters. Tab. I comprehends all other parameters. In this study, we do not analyze how these values should be set best but rather want to find the basic performance trends of the approach.

The awareness is measured every $100 \mathrm{msec}$. A neighbor is lost if no beacon was received for $750 \mathrm{msec}$. A forward request is triggered $50 \mathrm{msec}$ after the successive beacon interval. This copes for increased medium access delay [12] in high densities as well as clock drifts and processing delays. All potential forwarders randomly select an additional backoff from an interval of $10 \mathrm{msec}$.

\section{B. Simulation Results}

According to the metrics given in Sec. III, we evaluate the performance of our selective forwarding approach and compared it with different static beacon rates.

1) Quality of Cooperative Awareness: Fig. 4 shows the awareness for both, different beacon rates and the selective forwarding approach in low $(200-600$ vehicles $)$, medium $(800-1200$ vehicles) and in high density $(1600-2000$ vehicles). Note that for the results from the high density scenario of 1600 and 2000 vehicles there is only few movement due to many traffic jams. Hence, changes in the neighborhood occur more rarely.

From the static beacon rates, neither a low beacon rate $(2 \mathrm{~Hz})$ nor a medium beacon rate $(4 \mathrm{~Hz})$ provide a sufficiently high awareness quality throughout all density scenarios. For low vehicle densities, $4 \mathrm{~Hz}$ performs better than $2 \mathrm{~Hz}$ due to a lower latency of initially finding a new neighbor. However, as the density increases the increased packet collisions due twice the load decrease the awareness. From a density of 1200 vehicles on, $2 \mathrm{~Hz}$ provides even a better result of the awareness

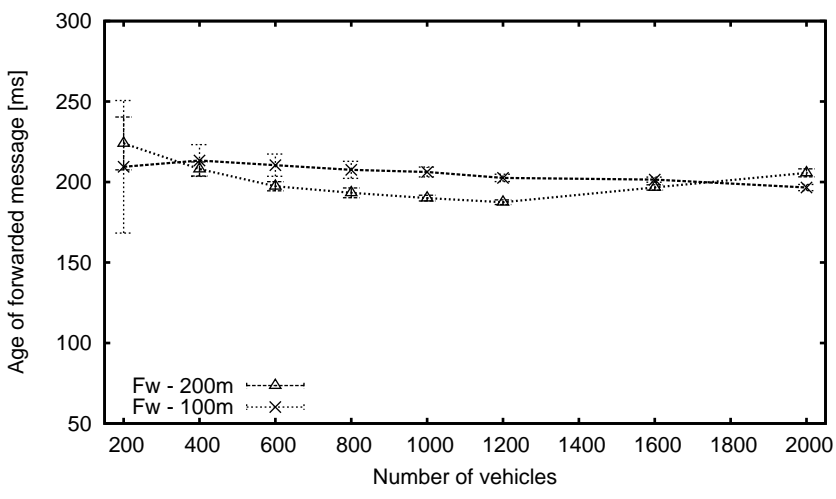

Fig. 5. Age of received information.

quality. For the sake of readability, we did not plot the results for $6 \mathrm{~Hz}, 8 \mathrm{~Hz}$, and $10 \mathrm{~Hz}$ as those results are much worse than $4 \mathrm{~Hz}$ in all tested scenarios.

The results for the static beacon rates show that the main task of the exchange of beacons, to establish cooperative awareness, is not sufficiently fulfilled in medium and especially not in high density scenarios. In medium density, the awareness is only at 70 to 85 percent in the applicationrelevant area. Even worse, in high density, the awareness is only at 25 to 40 percent. Increased packet loss at far distances beyond the communication range under interference is the cause for that in case of the highest beacon rate. Whereas for the lowest beacon rate, the awareness is lowered due to two reasons. First, the beacon rate is too low to cope with the dynamic changes in the topology. Once a vehicle $A$ comes into range of $B$, it takes a quarter of a second in the average case until $A$ transmits a beacon. Second, if there is even a collision and $B$ cannot receive the beacon, it takes another $500 \mathrm{msec}$ till $A$ transmits the next beacon. So, for static beacon rates, $4 \mathrm{~Hz}$ seems to be (close to) the global optimum of the awareness quality.

This also shows that the communication range under interference does not translate into reliable $100 \%$ awareness quality. Single packet loss still occurs which decreases the awareness dramatically in high densities, even in the applicationrelevant area of 300 meters around the transmitting vehicle. We identify this as room to improve the awareness, i.e. to bring it closer to $100 \%$.

Fig. 4 also shows the potential of the selective forward and its proper setup. With $d_{\text {comm }}=d_{a p p}=200$ meters it is possible to increase the awareness quality in medium density by nearly $20 \%$. We will also refer to this setup as most aggressive setup of the selective forwarding in the following. Comparing the figures of low and medium reveals that the selective forward performs even better with higher vehicle density. The significant benefit decreases to few percents for the setup of highest density.

For the complete discussion of the quality of cooperative awareness, we examine the average age of forwarded information in the following. Higher static beacon rates provide of course better up-to-date information than the forward, i.e. the age is negligible. 


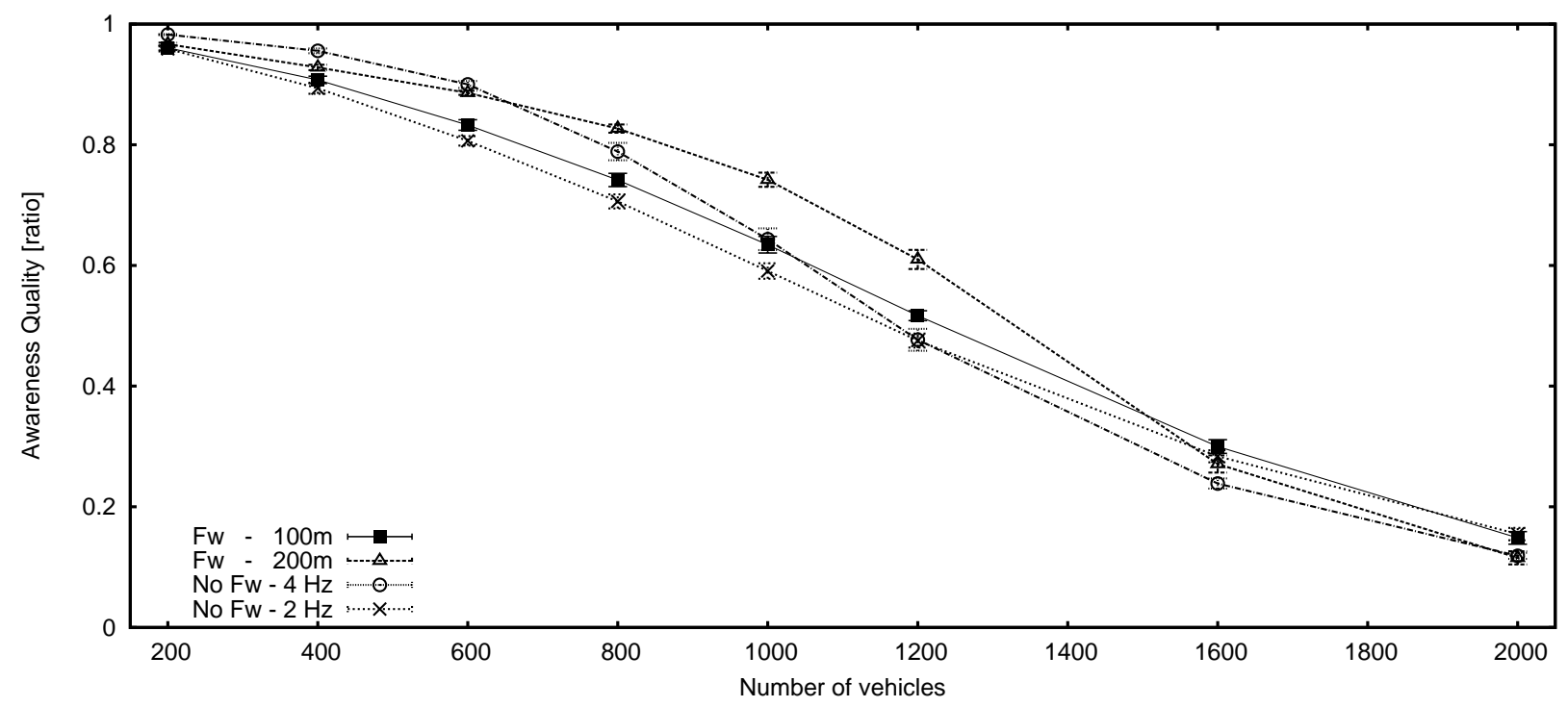

Fig. 4. Awareness in medium density a) without forwarding, b) with forwarding, and in high density c) without forwarding, d) with forwarding.

Fig. 5 illustrates the average age of all received forwarded beacons. Due to the piggybacking of beacon requests, the age of the forwarded beacon is coupled with the regular beacon interval, i.e. $500 \mathrm{msec}$. The result presented in the figure is thus as expected: Nearly constant in all densities, around 250 msec. As we assumed that vehicles start the beaconing uniformly distributed, this result matches the expectation. A lower average age can be achieved in higher densities and also by a higher value for $d_{a p p}$ and $d_{c o m m}$ as there are more potential requesters, increasing the probability to piggyback the request earlier. The age increases again beyond the density of 1200 vehicles. We observed increased collisions of the forwards due to increased size of the message (concatenation) and a too optimistic assumption on the communication range under interference. Another consequence of that is also a drop in primary benefit, discussed in the next subsection.

2) Efficiency and Cooperativeness: Fig. 6 compares the efficiency in channel usage between the forwarding approach and higher static beacon rates. A beacon rate of $2 \mathrm{~Hz}$ provides the best performance among the tested beacon rates and stays even with 2000 vehicles above 40 percent of success rate. Similarly, the success rate of $4 \mathrm{~Hz}$ drops linearly but below 20 percent. The highest beacon rate with $10 \mathrm{~Hz}$ shows the least success rate throughout all vehicle densities. But it can be seen that the success rate seems to stabilize at 10 percent, which is mainly due to successful receptions from vehicles within the reliable communication range.

The selective forwarding approach stays between 2 and $4 \mathrm{~Hz}$ in low and medium density but drops especially with the most aggressive setup of 300 meters quickly below the success rate of $4 \mathrm{~Hz}$ in higher densities. As we require a proper operation under high load situations, the most aggressive setup causes too much interference. So, we limit our approach to the setup of 200 meters for the remaining evaluation.

Fig. 7 shows how the vehicles that have not received the beacon directly have a benefit from the forward, i.e. primary

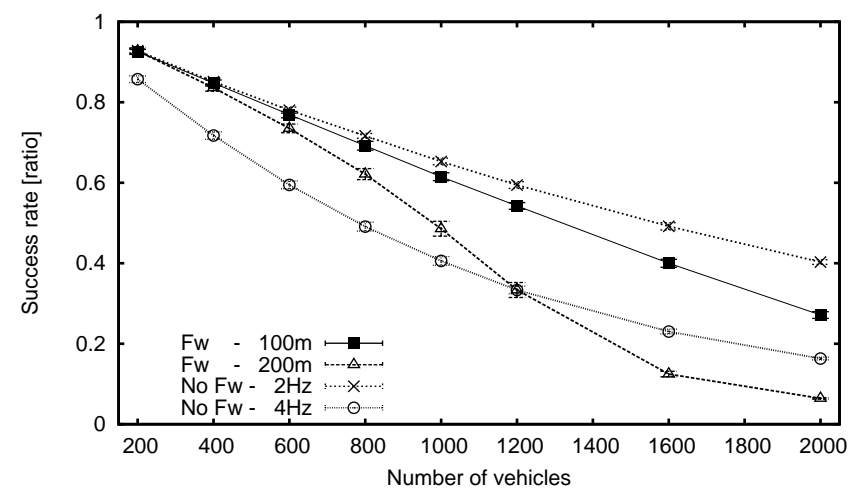

Fig. 6. Packet success rate, i.e. efficiency of the communication.

and secondary benefit. We refine the result of the primary benefit even further, by explicitly showing the primary benefit for vehicles within the safety-relevant area of 200 meters around the source. The total primary benefit is already at low densities above 10. Even the close primary benefit always stays always around or above two which means that per forwarded beacon at least two vehicles receive updated information on average. This ratio also confirms the increase of awareness, analyzed in Sec. V-B1. Beyond a density of 1000 vehicles, the total primary benefit decreases but the close primary benefit still increases. The reason for that is the higher likeliness of collisions of the forwarded beacon beyond but not in the communication range under interference. The increasing close primary benefit emphasizes that the usage of the communication range under interference scales w.r.t. well even in very high densities w.r.t. the benefit. We also observed a higher likeliness of concatenation, where two or more beacons are sent in one forward. It seems that the concatenation outperforms the increases packet error probability. It has to be investigated if splitting of the forward improves the result 


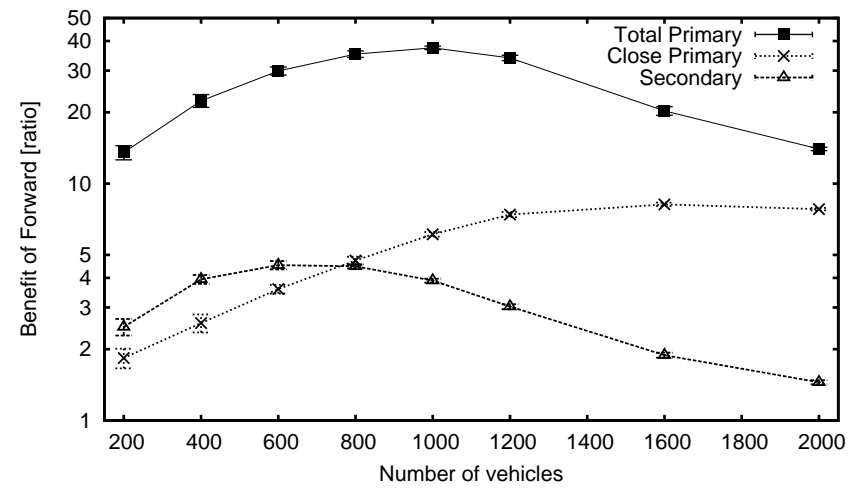

Fig. 7. Average Primary and secondary benefit of forward beacon, $d_{a p p}=$ $d_{\text {comm }}=200$ meters.

or rather reduces the benefit. However, the coordination with other potential forwarders is a difficult step then which can go at the expense of robustness. Redundant forwards may be a consequence of not properly overhearing a forward.

The figure also shows the secondary benefit which is much less than primary benefit in all scenarios. It increases similarly to the primary benefit in scenarios of medium density and then decreases in high densities. The secondary benefit is a beneficial byproduct of the selective forward but not the primary goal. Comparing both results shows that the selection strategy for forwarding is well-scoped to extending mainly the awareness within the safety-relevant area but not the two hop range.

\section{Summary}

The evaluation of the selective forward shows that the quality of cooperative awareness is can be increased especially under medium density scenarios. With a defensive setup of our approach to operate only in a range of 200 meters around the source of the respective beacon, the overhead is in the same order as for a beacon rate of $4 \mathrm{~Hz}$, except for very high density scenarios. We have also confirmed the cooperativeness of our approach. It provides remarkable primary benefit for each forwarded beacon. The secondary benefit improves the awareness beyond the one-hop communication range, as a minor beneficial byproduct. A comparison both emphasizes the careful selection of beacons to forward to update the awareness only within the application-relevant area. The ability to operate well in high load situations is confirmed, as the approach performs even better with higher vehicle density (up to a certain local maximum). The average age of forwarded information slightly decreases, whereas the degradation of the success rate stays moderate.

\section{CONCLUSiOns}

We discussed selective beacon forwarding to improve cooperative awareness. For this purpose, we established metrics to measure the quality of cooperative awareness and to evaluate the efficiency of beacon forwarding. We identified two of the main reasons for packet loss, interference and shadowing, which are particularly addressed by the forwarding.
The key strategy is that a beacon is forwarded by a vehicle that is close to the requesting vehicle. Supported by the communication range under interference, forwarded beacons are most likely to be successfully received by the addressed requesting vehicles which can significantly improve the cooperative awareness.

We identified that higher beacon rates do not provide a better awareness as the packet loss due to the channel overload dominates the awareness degradation. Selective forwarding is able to mitigate this degradation. By focusing selective forwarding appropriately, the increase of the channel load stays moderate and hence the degradation of packet success rate.

Our future work comprises a study on how to vary $d_{a p p}$ and $d_{\text {comm }}$ independently of each other and address a dynamically varying beacon rate [13]. Also, realistic fading and shadowing models need to be investigated to evaluate the benefit in compensating long-term shadowing between two neighbors.

\section{REFERENCES}

[1] ETSI TS 102 637-2, Intelligent Transport Systems (ITS); Vehicular Communications; Basic Set of Applications; Part 2: Specification of Co-operative Awareness Basic Service. ETSI, Sophia Antipolis Cedex, France, 2010.

[2] ETSI TS 102 637-3, Intelligent Transport Systems (ITS); Vehicular Communications; Basic Set of Application; Part 3: Specification of Decentralized Environmental Notification Basic Service. ETSI, Sophia Antipolis Cedex, France, 2010.

[3] R. K. Schmidt, T. Köllmer, T. Leinmüller, B. Böddeker, and G. Schäfer, "Degradation of Transmission Range in VANETS caused by Interference," PIK - Praxis der Informationsverarbeitung und Kommunikation (Special Issue on Mobile Ad-hoc Networks), vol. 32, pp. 224-234, 2009.

[4] B. Gallagher, H. Akalsuka, and H. Suzuki, "Wireless communications for vehicle safety: Radio link performance and wireless connectivity methods," IEEE Vehicular Technology Magazine, vol. 1, no. 4, pp. 424, 2006.

[5] T. K. Mak, K. P. Laberteaux, and R. Sengupta, "A multi-channel vanet providing concurrent safety and commercial services,' in VANET '05: Proceedings of the 2nd ACM international workshop on Vehicular ad hoc networks. New York, NY, USA: ACM, 2005, pp. 1-9.

[6] G. Korkmaz, E. Ekici, F. Özgüner, and U. Özgüner, "Urban multi-hop broadcast protocol for inter-vehicle communication systems," in VANET '04: Proceedings of the 1st ACM international workshop on Vehicular ad hoc networks. New York, NY, USA: ACM, 2004, pp. 76-85.

[7] S. Park and R. R. Palasdeokar, "Reliable one-hop broadcasting (rob) in mobile ad hoc networks," in PE-WASUN '05: Proceedings of the 2nd ACM international workshop on Performance evaluation of wireless ad hoc, sensor, and ubiquitous networks. New York, NY, USA: ACM, 2005, pp. 234-237.

[8] M. N. Mariyasagayam, T. Osafune, and M. Lenardi, "Enhanced multihop vehicular broadcast (mhvb) for active safety applications," in Telecommunications, 2007. ITST '07. 7th International Conference on ITS, 2007, pp. 1-6.

[9] J. Mittag, F. Thomas, J. Härri, and H. Hartenstein, "A comparison of single- and multi-hop beaconing in vanets," in VANET '09: Proceedings of the sixth ACM international workshop on VehiculAr InterNETworking. New York, NY, USA: ACM, 2009, pp. 69-78.

[10] S. Yousefi, S. Bastani, and M. Fathy, "Performance of beacon safety message dissemination in vehicular ad hoc networks (vanets)," in Journal of Zhejiang University - Science A. Springer-Verlag GmbH, Oct. 2008, pp. 1990-2004.

[11] ETSI, Intelligent Transport Systems (ITS); Vehicular Communications; Basic Set of Applications; Definitions (ETSI TR 102638 V1.1.1), European Telecommunications Standards Institute, Jun. 2009.

[12] R. K. Schmidt, T. Leinmüller, and G. Schäfer, "Adapting the Wireless Carrier Sensing for VANETs," in 6th International Workshop on Intelligent Transportation (WIT), Hamburg, Germany, March 2010.

[13] R. K. Schmidt, T. Leinmüller, E. Schoch, F. Kargl, and G. Schäfer, "Exploration of Adaptive Beaconing for Efficient Intervehicle Safety Communication," IEEE Network Magazine, Special Issue on Advances in Vehicular Communications Networks, vol. 24, pp. 14 - 19, 2010. 